\title{
SEDIMENT TRANSPORT AT THE BOTTOM OF SEA WAVES
}

\author{
G. Vittori ${ }^{1}$, P. Blondeaux ${ }^{1}$
}

\begin{abstract}
The flow in the wall boundary layer generated close to the sea bottom by the propagation of a monochromatic surface wave is determined by considering small values of both the wave steepness and the ratio between the thickness of the boundary layer and the local water depth. Depending on the hydrodynamic conditions, the sea bottom can be plane or rippled. The geometrical characteristics of the bottom forms are predicted using empirical formulae and, then, the bedforms are assumed to behave as a bottom roughness, the size of which is related to the size of the ripples. The bottom boundary layer is assumed to be turbulent and the flow field is computed by means of a two-equation turbulence model. Then the sediment transport is evaluated. The bed load is obtained using an empirical relationship. The suspended load is determined by computing the sediment flux, once the spatial and temporal distribution of sediment concentration is determined. A comparison of the model findings with the experimental results supports the approach.
\end{abstract}

Keywords: sea waves, steady streaming, turbulence modeling, sediment transport.

\section{INTRODUCTION}

To obtain reliable estimates of the sediment transport rate in the coastal region and to predict erosion and deposition processes, it is necessary to have a detailed knowledge of the flow within the bottom boundary layer which is generated by propagating surface waves and, in particular, to take into account nonlinear effects. When a regular wave moves into shallow water, nonlinear effects in the bottom boundary layer produce a steady streaming and a net sediment transport which become significant in the nearshore region when waves attain large amplitude. Longuet-Higgins (1953) studied the boundary layer at the sea bottom due to the passage of surface waves and determined the steady streaming by considering the laminar regime and a flat bottom. Experimental measurements show that significant departures from the predictions obtained by means of Longuet-Higgins' analysis may occur. Collins (1963) observed a deviation from the Longuet-Higgins' (1953) results and assumed it is induced by the presence of turbulence. Of course also the presence of ripples, which cause boundary layer separation (Blondeaux \& Vittori, 1991a,b), affects the steady streaming and, in turn, the steady streaming affects ripple dynamics (Foti \& Blondeaux ,1995; Vittori \& Blondeaux, 1996; Blondeaux, Vittori \& Foti, 2000).

Different approaches can be used to describe the boundary layer generated by sea waves when the flow regime is turbulent. Early studies (e.g. Kajiura,1968; Trowbridge \& Madsen, 1984a,b) prescribed the eddy viscosity as a given function of the distance from the bottom and time. Though widely used for their simplicity, such approximations are rigorously founded only when turbulence is in local equilibrium and turbulence advection and diffusion are not important. Therefore, later, the study of the boundary layer under sea waves was tackled also using more sophisticated two-equation turbulence models such as the k- $\varepsilon$ model (e.g. Justesen, 1988) or Saffman's model (e.g. Blondeaux, 1987). The mass transport in a turbulent boundary layer was first determined by Longuet- Higgins (1958) by means of a simple approach. The mass transport velocity at the outer edge of the boundary layer was found by Longuet-Higgins (1958) to be unaffected by the presence of turbulence. More recently, Chowdhury, Sato \& Ueno (1997) evaluated the mean flow within the bottom boundary layer by considering a closure scheme based on a one-equation turbulence model. They made a detailed analysis of the mean velocity profile and showed that it points in the offshore direction for relatively long waves, in accordance with previous investigations. Moreover, they found that the thickness of the boundary layer gradually increases when the roughness of the bed increases. As pointed out by Chowdhury et al. (1997), the differences of the results obtained with the use of different eddy viscosity models show that it is preferable to adopt sophisticated models based on transport equations for turbulence characteristics such as two-equation turbulence models.

A large number of theoretical investigations and experimental works consider an oscillatory boundary layer, where the velocity is uniform in the flow direction, as a prototype of the boundary layer at the bottom of sea waves. In particular, to reproduce the hydrodynamics and sediment transport generated by sea waves close to the bottom, oscillating water tunnels are used (Van der Werf et al.

1 DICCA, University of Genoa, via Montallegro 1, Genova, 16145, Italy 
2009). However, as recently pointed out by Gonzalez-Rodriguez \& Madsen (2011), the actual flow at the bottom of sea waves depends on the coordinate $x^{\circ}$ in the direction of wave propagation and this spatial dependence has a significant influence on the steady streaming and the sediment transport.

Recently, Blondeaux et al. (2012) have focussed their attention in the bottom boundary layer under a propagating sea wave and have determined the flow and the sediment transport by assuming that the sea bottom is flat. Their results show that the steady velocity component and the net (wave averaged) sediment transport are different from those generated by asymmetrical fluid oscillations in an oscillating water tunnel (U-tube) even if the values of the significant parameters (amplitude and period of the fluid oscillations, asymmetry index) are the same. The analysis of Blondeaux et al. (2012) considers large values of the Shields parameter such that the small scale bedforms (ripples), which are usually generated by the interaction of sea waves with a cohesionless sandy bottom, are washed out and the bottom can be assumed to be flat. Even though large values of the Shields parameter are usually found in the nearshore region during storms, for mild weather conditions the shoaling region is characterized by moderate or relatively small values of the Shields parameter such that the sea bottom is rippled (Sleath, 1984). Motivated by the finding of Chowdhury et al. (1997) on the relevant effect of bottom roughness on the bottom boundary layer, presently, the analysis of Blondeaux et al. (2012) is extended to take into account the effect of the presence of ripples on the steady streaming.

In the next section a brief description of the model is given. For the sake of clarity, the description is divided into a hydrodynamic part and a sediment transport part. The last part of the next section describes the numerical solution as well as the validation of the model. Then the description of the results and the conclusions follow.

\section{THE MODEL}

\section{The flow field}

A two-dimensional monochromatic surface gravity wave propagating in water of constant depth $h_{0}^{*}$ is considered. The sea bottom is made of sand characterized by a uniform grain size $d^{*}$ and density $\rho_{s}^{*}$. Hereinafter, a star denotes a dimensional quantity while the same symbol without the star denotes its dimensionless counterpart. Let us denote with $T^{*}=2 \pi / \omega^{*}$ and $L^{*}=2 \pi / k^{*}$ the period and the length of the wave, $\omega^{*}$ and $k^{*}$ being its angular frequency and wavenumber, respectively. Finally, let us introduce a Cartesian coordinate system $\left(x^{*}, y^{*}, z^{*}\right)$, with the $\left(x^{*}, z^{*}\right)$-plane coincident with the bottom, the $x^{*}$-axis being along the direction of wave propagation and pointing in the offshore direction and the $y^{*}$-axis pointing upwards. The free surface displacement associated with the wavetrain is described by

$$
y^{*}=h_{0}^{*}+\frac{a^{*}\left(x^{*}\right)}{2}\left[e^{i\left(k^{*} x^{*}+\omega^{*} t^{*}\right)}+\text { c.c. }\right]+\text { h.o.t }
$$

where $t^{*}$ is time, c.c. denotes the complex conjugate of a complex quantity and h.o.t. denotes higherorder terms. The amplitude of the wave $a^{*}\left(x^{*}\right)$ is assumed to depend on the coordinate $x^{*}$ because of the damping of the wave amplitude. Let us introduce the following dimensionless quantities

$$
\begin{aligned}
& (x, y)=\frac{\left(x^{*}, y^{*}\right)}{L^{*}}, \quad t=t^{*} \omega^{*}, \quad \eta=\frac{\eta^{*}}{a_{0}^{*} / S} \\
& (u, v)=\frac{\left(u^{*}, v^{*}\right)}{a_{0}^{*} \omega^{*} / S}, \quad p=\frac{p^{*}}{\rho a_{0}^{*}\left(\omega^{*}\right)^{2} L^{*} / S}
\end{aligned}
$$

where $\rho^{*}$ is water density, $\left(u^{*}, v^{*}\right)$ are the velocity components along the $\mathrm{x}^{*}$ - and $\mathrm{y}^{*}$-axes, respectively, $p^{*}$ is the pressure, $a_{0}^{*}$ is a measure of the wave amplitude of the incident wave and $S$ is equal to $\sinh \left(2 \pi h_{0}^{*} / L^{*}\right)$. The problem of flow determination is posed by continuity and momentum equations along with the kinematic and dynamic boundary conditions at the free surface and at the bottom. The form of the stress tensor depends on the flow regime which might be different in the 
boundary layer and in the region far from the bottom. Indeed turbulence is usually present in the bottom boundary layer, where the variables should be intended as the Reynolds-averaged quantities, but it vanishes moving far from the sea bed. A two-equation RANSE model is used to compute the turbulent flow-field thereby the eddy viscosity is introduced to quantify the Reynolds stresses inside the bottom boundary layer. The eddy viscosity of course vanishes moving far from the bottom.

Because of viscous effects, the amplitude of the sea wave is expected to decay on a spatial scale $\left(L^{*}\right)^{2} / \delta^{*}$ (Mei 1989), where $\delta^{*}=\sqrt{2 v^{*} / \omega^{*}}$ is the conventional thickness of the viscous boundary layer, $v^{*}$ being the kinematic viscosity of the fluid. Hence, let us introduce the new slow variable

$$
\chi=x \frac{\delta^{*}}{L^{*}}
$$

such that:

$$
a=\frac{a^{*}}{a_{0}^{*}}=a(\chi) .
$$

The resulting dimensionless problem is characterized by the following parameters:

$$
\varepsilon=\frac{a_{0}^{*}}{L^{*} S}, \quad \delta=\frac{\delta^{*}}{L^{*}}
$$

In the present study we consider a small-amplitude wave propagating over intermediate depths, such that $a_{0}^{*} \ll L^{*}$ and $\delta^{*} \ll L^{*}$. Therefore we assume:

$$
\varepsilon=\frac{a_{0}^{*}}{L^{*} S}<<1, \quad \delta=\frac{\delta^{*}}{L^{*}}<<1
$$

It is worthwhile to mention that the value of $\delta$ is related to $\varepsilon$ by:

$$
\delta=\frac{2 \varepsilon}{R_{\delta}} \quad \text { with } \quad R_{\delta}=\frac{\left(a_{0}^{*} \omega^{*} / S\right) \delta^{*}}{v^{*}}
$$

where $R_{\delta}$ is the Reynolds number formed with the amplitude of the velocity oscillations close to the bottom $\left(a_{0}^{*} \omega^{*} / S\right)$, the conventional thickness of the bottom boundary layer $\left(\delta^{*}\right)$ and the viscosity of the fluid. The Reynolds number $\operatorname{Re}=\left(U_{0}^{*}\right)^{2} /\left(v^{*} \omega^{*}\right)$, which is often used in coastal engineering, is simply $\operatorname{Re}=R_{\delta}^{2} / 2$.

The solution is determined for small values of the wave steepness $a_{0}^{*} / L^{*}$ and finite values of the Reynolds number $R_{\delta}$. Because of the finite value of $R_{\delta}$ and our interest in the bottom boundary layer, the solution is expanded in terms of $\delta$ instead of $\varepsilon$

$$
(u, v, p, \eta)=\left(U_{0}, V_{0}, P_{0}, \eta_{0}\right)+\delta\left(U_{1}, V_{1}, P_{1}, \eta_{1}\right)+O\left(\delta^{2}\right) .
$$

In the irrotational turbulence-free region (core region), both at the leading order of approximation and at second order, viscous and turbulence effects can be neglected. The solution follows closely Vittori \& Blondeaux(1996). In particular, at order $\delta$ the solution is composed of a steady part, independent of $x^{*}$, and a propagating part with angular frequency $2 \omega^{*}$. Both components are generated by non-linear effects. A third contribution is characterized by the angular frequency $\omega^{*}$ and is forced by the decay of the wave amplitude as it propagates. As the computed solution does not include viscous effects, boundary layers at the sea bottom and at the sea surface should be introduced. Presently 
attention is focused on the bottom boundary layer where the magnified variables

$$
(\tilde{x}, \tilde{y})=\frac{(x, y)}{\delta}
$$

are introduced together with a new time variable:

$$
\tilde{t}=2 \pi x+t
$$

necessary to simplify the algebra which is necessary to study the near-bed region.

The boundary conditions, to be applied in the boundary layer region, force the no-slip at the bottom and the matching of the solution with that describing the inviscid flow in the core region. The eddy viscosity, which is computed by using Saffman's (1970) turbulence model, is assumed to be a function of two turbulence local properties namely the pseudo-energy $e^{*}$ and the pseudo-vorticity $\Omega^{*}$, which satisfy non-linear diffusion equations. The values of the constants of the model are fixed following Saffman \& Wilcox (1974). The boundary conditions which complement the equations of the turbulence model are the vanishing of both $e^{*}$ and $\Omega^{*}$ in the inviscid region while, at the sea bottom, the turbulent kinetic energy vanishes and the pseudo-vorticty depends on the roughness parameter $z_{w}=z_{r}^{*} u_{\tau}^{*} / v^{*}$ through a universal function $\mathrm{F}\left(z_{w}\right), z_{r}^{*}$ and $u_{\tau}^{*}$ being the dimensional roughness size and friction velocity, respectively ( Blondeaux \& Colombini, 1985). The roughness size is related to sediment diameter if the sea bottom is plane $\left(z_{r}^{*}=2 d^{*}\right)$ and, following Van Rijn(1991), to the ripple height $\eta_{r}^{*}\left(z_{r}^{*}=\eta_{r}^{*}\right)$ for rippled beds. The ripple height is predicted, based on local conditions, by using the ripple predictor of Soulsby \& Whitehouse (2005)

\section{The sediment transport}

Once the hydrodynamic problem is solved, the dimensionless total sediment transport rate $Q$ can be computed considering both the bedload rate $\left(Q_{b}\right)$ and the suspended load rate $\left(Q_{s}\right)$. The dimensionless bed load rate $Q_{b}=Q_{b}^{*} / \sqrt{\left(\rho_{s}^{*} / \rho^{*}-1\right) g^{*}\left(d^{*}\right)^{3}}$ is evaluated by means of an empirical predictor. Presently the formula proposed by Fredsoe \& Deigaard(1992) is employed which reads:

$$
Q_{b}=5 p\left(\sqrt{\theta}-0.7 \sqrt{\theta_{c}}\right)
$$

where the probability $p$ that all the particle in a single layer will be in motion is computed by:

$$
p=\left[1+\left(\frac{\frac{1.51 \pi}{6}}{\theta-\theta_{c}}\right)^{4}\right]^{-(1 / 4)}
$$

and the Shields parameter $\theta$ is defined as:

$$
\theta=\frac{\tau^{*}}{\left(\rho_{s}^{*}-\rho^{*}\right) g^{*} d^{*}}
$$

In (12)-(13) $\tau^{*}$ is the bottom shear stress and $\theta_{c}$ is the critical value of the Shields parameter for the initiation of sediment motion. The dimensionless sediment suspended rate is computed by integrating the sediment flux from the reference level $\tilde{y}_{\text {ref }}^{*}$ up to the inviscid region and neglecting the diffusion contribution 


$$
Q_{s}=\frac{Q_{s}^{*}}{\sqrt{\left(\rho_{s}^{*} / \rho^{*}-1\right) g^{*}\left(d^{*}\right)^{3}}}=\frac{\psi_{d}}{d} \int_{\tilde{y}_{r e f}}^{\infty} u c d \tilde{y}
$$

where $\psi_{d}$ is the sediment mobility number defined by:

$$
\psi_{d}=\frac{\left(a_{0}^{*} \omega^{*} / S\right)^{2}}{\left(\rho_{s}^{*} / \rho^{*}-1\right) g^{*} d^{*}}
$$

and $d=d^{*} / \delta^{*}$ is the dimensionless grain size.

The sediment concentration $c$, appearing in (14), is derived from the mass balance equation, assuming that the sediment is transported by advection and by turbulent diffusion. Since the sediment concentration rapidly decays when moving far from the bottom, the sediment concentration is computed only in the bottom boundary layer, by solving the appropriate advection-diffusion equation where the fall velocity $w_{s}^{*}$, which depends on the sediment Reynolds number $R_{p}=\sqrt{\left(\rho_{s}^{*} / \rho^{*}-1\right) g^{*}\left(d^{*}\right)^{3}} / v^{*}$ appears. The boundary conditions to be forced for the sediment concentration are the vanishing of $c$ far from the bottom and the empirical relationship by Zyserman \& Fredsoe (1994) which prescribes the value of the sediment concentration at an assigned distance from the bottom as a function of the Shields parameter $\theta$. The equation for the sediment concentration is solved, similarly to momentum equation, by expanding $c$ in power series of $\delta$.

\section{The numerical solution}

The problems obtained at the different orders of approximation in $\delta$, are solved numerically. The momentum and sediment concentration equations are solved by means of a Runge-Kutta method of second order to advance in time, while a finite difference approach is used to approximate spatial derivatives in the $\tilde{y}$-direction. To increase the accuracy of the solution close to the bottom, where the gradients are larger, the grid-points have been clustered close to the bottom. The numerical procedure has been validated by comparing the obtained results with experimental data obtained for a flat bed, both in wave channels and in U-tubes. More details on the formulation of the problem and the numerical approach can be found in Blondeaux et al. (2012).

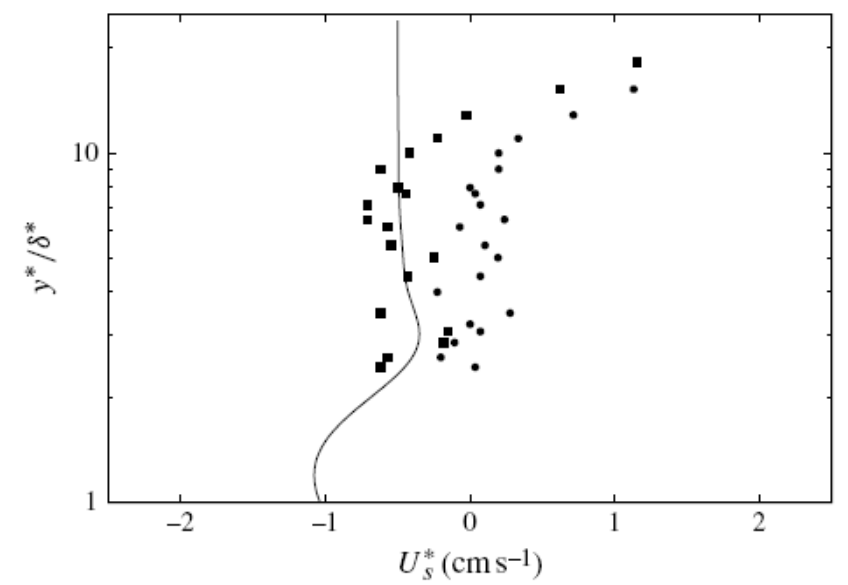

Figure 1: Comparison between the steady velocity profile under surface waves (solid line) and the experimental data of Van Doorn (1981). Black squares are the velocity measurements above the roughness crests and the dots are the velocity measurements above the roughness troughs.

A comparison of the computed values of the streamwise steady velocity with the experimental results obtained by Van Doorn (1981) in a wave channel is shown in figure 1. Hereinafter, negative values of the steady velocity component are meant to be in the direction of wave propagation. The parameters of the model are fixed considering a wave amplitude equal to $5.2 \mathrm{~cm}$, a wave period equal to $2 \mathrm{~s}$, a water depth of about $30 \mathrm{~cm}$ and a regular roughness characterized by a size equal to $2.1 \mathrm{~cm}$. Van Doorn (1981) measured the velocity profile in two positions: above the crest of a roughness element 
and in the middle between the crests of two roughness elements. Close to the bottom, a fair agreement between the numerical results and the average of the measurements above the crests and troughs of the roughness elements is found and supports the present model. Moreover, the present predictions are closer to the experimental data than those obtained by previous researchers (e.g. Trowbridge \& Madsen 1984b). The significant differences which appear far from the bottom might be due to the finite length of the wave channel used in the experiments and the consequent offshore steady current which is present outside the boundary layer.

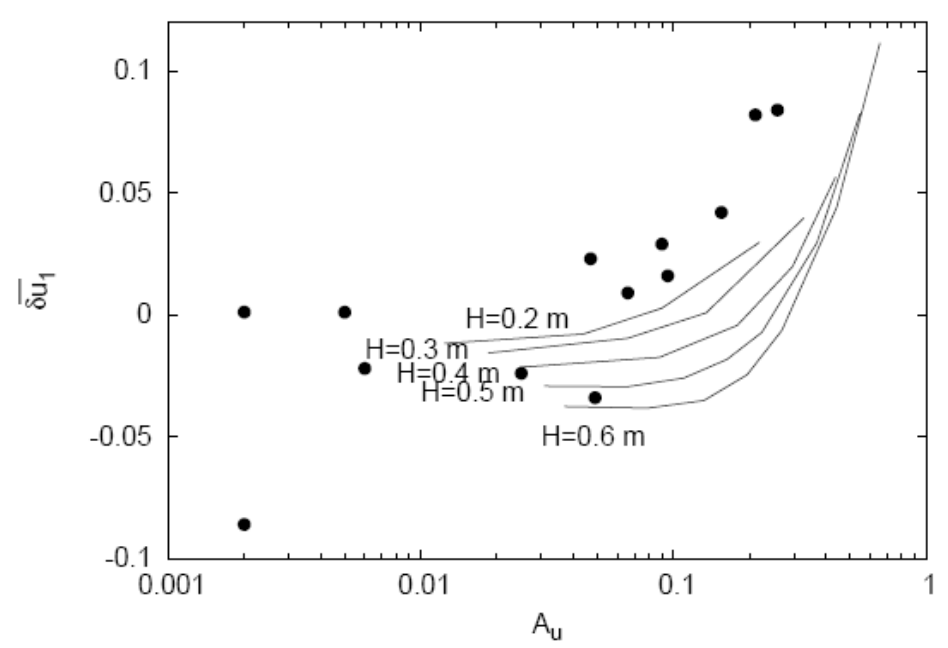

Figure 2. Time averaged values of the dimensionless velocity $\left(\overline{\delta u_{1}}\right)$ just outside the bottom boundary layer plotted versus the asymmetry index $\boldsymbol{A}_{u}$. Continuous line, theoretical values for $h_{0}^{*}=2.2 \mathbf{m}, \boldsymbol{T}^{*}$ ranging between 3 and $8 \mathrm{~s}$ and different values of $H^{*}$ (wave height); dots, experimental measurements Scandura \& Foti (2011)

Figure 2 shows the value of $\delta \bar{u}_{1}$, an overbar indicating time-average, at the top of the boundary layer, plotted versus the asymmetry index $A_{u}$ defined by Scandura \& Foti (2011) as

$$
A_{u}=\frac{U_{\max }^{*}-U_{\min }^{*}}{U_{\max }^{*}+U_{\min }^{*}}
$$

where $U_{\max }^{*}$ and $U_{\min }^{*}$ are the maximum values of the irrotational velocity close to the bottom in the direction of wave propagation and in the opposite direction, respectively. The data shown in figure 2 are obtained from figure 9 of the paper of Scandura \& Foti (2011) which does not allow to identify all the parameters of a single experiment. It is worthwhile to mention that, during Scandura \& Foti's experiments, ripples formed. The present results are obtained by fixing the water depth equal to (the experimental value) $2.2 \mathrm{~m}$, by considering wave heights $\mathrm{H}^{*}$ equal to $0.2,0.3,0.4,0.5$ and $0.6 \mathrm{~m}$ and by varying the wave period within the range $(3,8) \mathrm{s}$. From these dimensional quantities, the dimensionelss parameters introduced in our analysis, namely the Reynolds number $R_{\delta}$, the wave steepness $\varepsilon$ and the ratio $h^{*} / L^{*}$, can be easily obtained. The roughness related to the ripples is fixed by using Soulsby \& Whitehouse's predictor. Both numerical predictions and the laboratory data show that for small values of $A_{u}$, the intensity of turbulence during the onshore and offshore phases of the wave cycle are similar in such a way that the mechanism described by Longuet-Higgins (1953) prevails and the steady velocity component is negative, i.e. onshore directed. Then, the steady velocity component increases if the value of $A_{u}$ is increased and the steady streaming reverses its direction when $A_{u}$ becomes larger than a threshold value which depends on $H^{*}$. Even though the theoretical analysis seems to underestimate the steady velocity component for large values of $A_{u}$, the agreement between the theoretical results and the experimental measurements is fair, also taking into account the scatter of the laboratory data. More validation tests can be found in Blondeaux et al. (2012). 


\section{RESULTS AND DISCUSSION}

\section{Plane bed}

As already pointed out, the boundary layer generated by sea waves close to the bottom is often approximated by the boundary layer generated by the uniform oscillations of a fluid close to a wall. Such an approach well describes the actual flow field at the leading order of approximation, but it does not provide the correct description of the flow at the second-order of approximation, since the velocity field is characterized by a significant $\tilde{x}$-dependence. To ascertain what is the degree of accuracy in the modeling of the actual boundary layer by means of the uniform flow approximation, it is necessary to compare the results of the model for the wave case with those obtained for the uniform case (U-tube case), obtained from the model by switching off the $\tilde{x}$-dependence. Even though the two cases do not differ at the leading order of approximation, the steady streaming originated in a U-tube is different from that generated at the bottom of sea waves. Moreover, the former does not depend significantly on the wave period $T^{*}$. Indeed, a variation of $T^{*}$ induces a variation of the value of the Reynolds number. However, an analysis of the momentum equations for the U-tube case shows that the equations do not depend on $R_{\delta}$ both at the leading order of approximation and at order $\delta$ and the influence of $R_{\delta}$ on turbulence dynamics is not so strong to give rise to significantly different values of $\bar{u}_{1}$ (an overbar denotes a wave-averaged quantity). Figure 3 shows the time-averaged (wave-averaged) velocity profile close to the bottom for fixed values of $U_{r m s}^{*}, T^{*}$ and $A_{u}$ and for both a uniform flow (broken line) and an actual sea wave propagating in water of constant depth (solid line). The parameter $U_{r m s}^{*}$ is defined as:

$$
U_{r m s}^{\star}=\sqrt{\frac{\left(U_{\max }^{\star 2}+U_{m i n}^{\star 2}\right)}{8}}
$$

To allow an easy comparison of the results, the figure shows the dimensionless value of $\bar{u}_{1}$ multiplied by $\delta$. Even though the values of $U_{r m s}^{*}, T^{*}$ and $A_{u}$ are the same for the U-tube and wave cases, the U-tube case leads to values of the steady streaming which are qualitatively different from those found for an actual wave. It is worth pointing out that $U_{r m s}^{*}=0.6 \mathrm{~m} / \mathrm{s}, A_{u}=0.2, T^{*}=7 \mathrm{~s}$ and $T^{*}=10 \mathrm{~s}$ correspond to waves characterized by amplitudes of about $1.1,1.3 \mathrm{~m}$, wavelengths of about $47.9,89.7$ $\mathrm{m}$, propagating in water of constant depth equal to about 5.6 and $9.3 \mathrm{~m}$, respectively.

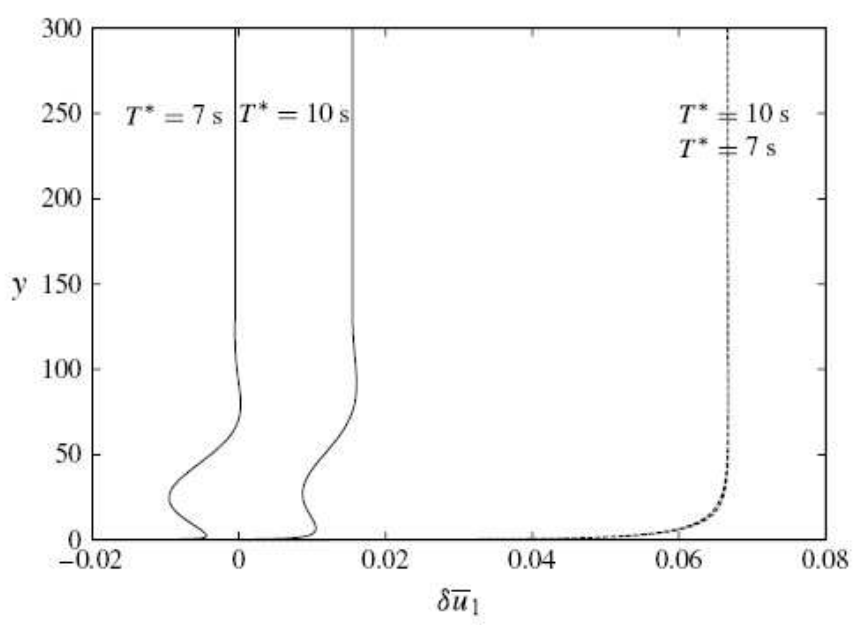

Figure 3. Dimensionless steady velocity component generated by an oscillatory boundary layer in a wave tunnel (broken line) and at the bottom of sea waves (solid lines) for two different values of $T^{\star}$ and a flat bed $\left(U_{r m s}^{*}=0.6 \mathrm{~m} / \mathrm{s}, A_{u}=0.2, d^{*}=0.13 \mathrm{~mm}\right)$.

Figure 4 shows the dimensionless steady velocity component at the upper edge of the wave 
boundary layer for fixed values of $U_{r m s}^{*}, A_{u}$ and $d^{*}$ as function of $T^{*}$. The results show negative mean velocities for the small wave periods which correspond to relatively short waves. For relatively long periods and long waves, the steady streaming reverses its direction and points in the offshore direction, which is one of the noticeable features of the turbulent wave boundary layer. The corresponding mean velocity profiles for the different values of $T^{*}$ are plotted in figure 5 . The modulus of the velocity is characterized by different relative maxima. The first maximum takes place close to the bottom and cannot be easily appreciated in figure 5. Then, for relatively small values of $T^{*}$, the largest value of the mean velocity is observed when $y^{*}$ falls between $20 \delta^{*}$ and $25 \delta^{*}$ and points in the onshore direction. For relatively large values of $T^{*}$, the maximum values of the mean velocity take place far from the bottom and point in the offshore direction.

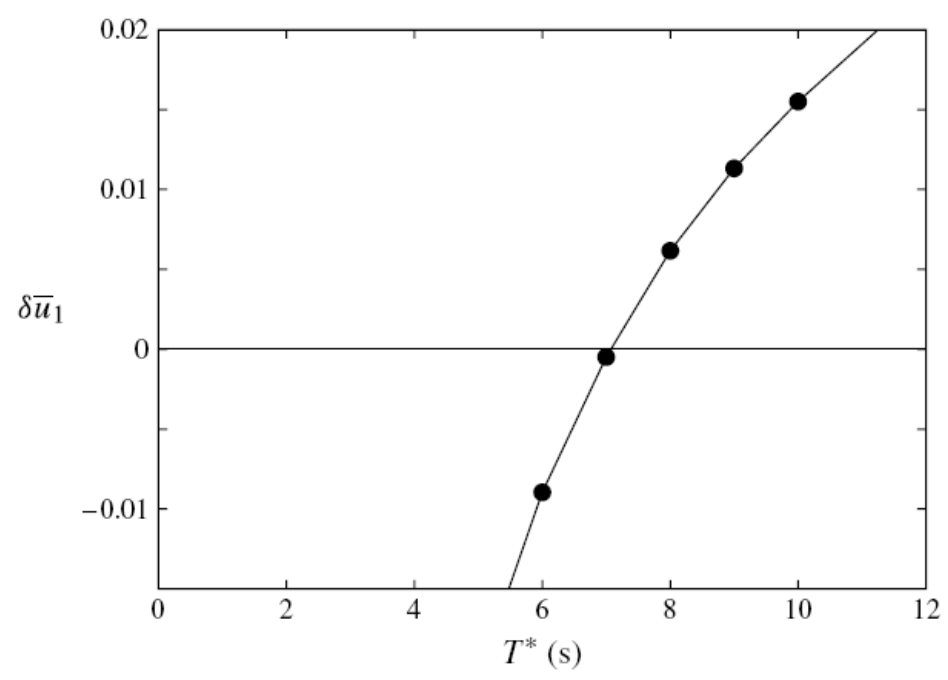

Figure 4. Dimensionless steady velocity component generated by surface waves at the edge of the boundary layer, plotted versus $T^{*}$ for a flat bed $\left(U_{r m s}^{*}=0.6 m s^{-1}, A_{u}=0.2, d^{*}=0.13 \mathrm{~mm}\right)$.

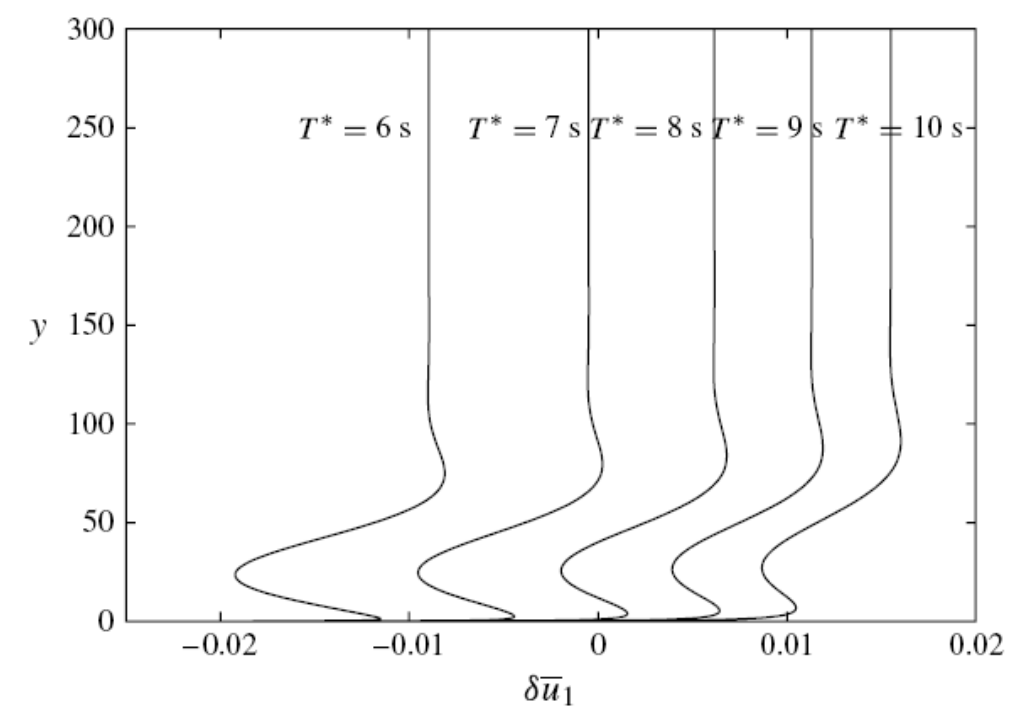

Figure 5. Dimensionless steady velocity component generated by surface waves in the bottom boundary layer, plotted versus y for different values of $\boldsymbol{T}^{*}$ and a flat bed $\left(U_{m s}^{*}=0.6 \mathrm{~ms}^{-1}, \boldsymbol{A}_{\boldsymbol{u}}=\mathbf{0 . 2}\right.$, $\left.d^{*}=\mathbf{0 . 1 3} \mathbf{~ m m}\right)$.

Notwithstanding the steady streaming reverses its direction as $T^{*}$ is increased, the net sediment transport rate always points onshore (see figure 6). As already pointed out, the sediment transport rate is 
given by the sum of the bed load $\left(Q_{b}\right)$ and the suspended load $\left(Q_{s}\right)$. However, in the range of the parameters presently investigated, the wave-averaged value of $Q_{s}$ turns out to be much larger than the wave averaged value of $Q_{b}$, which provides a negligible contribution to $\bar{Q}$. For the parameters shown in figure 6, the time-averaged suspended sediment flux turns out to be negative both close to the bottom and moving far from it, thus inducing a net onshore sediment transport. It is worth pointing out that $U_{r m s}^{*}=0.6 \mathrm{~ms}^{-1}, A_{u}=0.2$ and values of $T^{*}$ ranging between 6 and $10 \mathrm{~s}$ correspond to waves characterized by amplitudes ranging between 1.0 and $1.3 \mathrm{~m}$, wavelengths ranging between 36.5 and $89.7 \mathrm{~m}$, propagating in water of constant depth ranging between 4.5 and $9.3 \mathrm{~m}$.

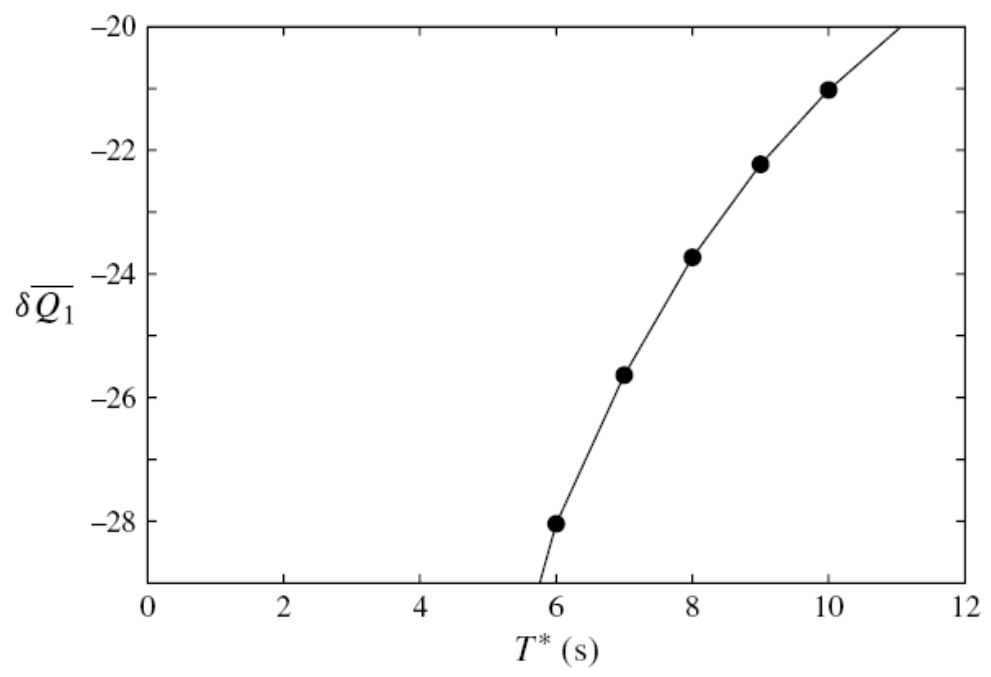

Figure 6. Dimensionless time averaged total sediment transport rate generated by surface waves, plotted versus $T^{*}$ for a flat bed $\left(U_{m s}^{-}=0.6 m s^{-1}, A_{u}=\mathbf{0 . 2}, d^{*}=\mathbf{0 . 1 3} \mathbf{m m}\right)$.

A detailed study has been carried out also on the influence of the other dimensionless parameters, namely $A_{u}, U_{r m s}^{*}$ and $d^{*}$. The results, obtained for $T^{*}=7 \mathrm{~s}, U_{r m s}^{*}=0.6 m s^{-1}$ and $d^{*}=0.13 \mathrm{~mm}$, show that for strongly skewed waves (large values of $A_{u}$ ), the steady velocity component is offshore directed while a weak skewness gives rise to an onshore directed steady streaming. Even though, for large values of $A_{u}$, the steady streaming points in the offshore direction, the time-averaged suspended sediment flux points in the onshore direction showing, once more, that the sign of the sediment transport cannot be inferred from the sign of the steady velocity component. The results, obtained for $A_{u}=0.2, T^{*}=7 \mathrm{~s}$ and $d^{*}=0.13$ $\mathrm{mm}$, show that an increase of the amplitude of the velocity oscillations $\left(U_{m s}^{*}\right)$ close to the sea bed causes the steady streaming to reverse its direction. Indeed, for relatively low values of $U_{m s}^{*}$ the steady velocity component points in the offshore direction while for relatively high values of $U_{m s}^{*}$ the steady velocity component points in the onshore direction. Even though for small values of $U_{m s}^{*}$ the steady velocity component points in the offshore direction, the time-averaged sediment transport rate is always negative (onshore directed). Of course, the sediment transport rate decreases as the grain size increases. Moreover, even though the sediment transport rate is found to be always dominated by the suspended load, the ratio between the bed load and the suspended load increases as $d^{*}$ increases. However, the sediment transport rate is always negative, i.e. it points always in the onshore direction. Positive values of the sediment transport rate have never been found in the range of the parameters such that the bottom is flat.

\section{Rippled bed}

Figure 7 shows the dimensionless steady velocity component at the upper edge of the wave boundary layer for fixed values of $A_{w}$, and $d^{*}$ as function of $T^{*}$ for three different values of $U_{r m s}^{*}$. The values of the parameters are such that ripples are generated. The ripple characteristics are determined by means of Soulsby \& Whitehouse's (2005) predictor and are given in Table 1. It is worthwhile to 
notice that for the smallest value of $U_{r m s}^{*}$, both ripple length and height first increase and then decrease as $T^{*}$ is increased, while they decrease for increasing values of $T^{*}$ if larger values of $U_{r m s}^{*}$ are considered. For $U_{r m s}^{*}=0.25 \mathrm{~m} / \mathrm{s}$, the results show positive (offshore directed) mean velocities for small wave periods. If the period of the waves is increased, first, the offshore steady streaming increases but it reaches a maximum for $T^{*}$ ranging in $(8,9) \mathrm{s}$ and a further increase of the period leads to a decrease of the steady velocity component and then to flow reversal. For $U_{r m s}^{*}=0.15 \mathrm{~m} / \mathrm{s}$ the steady velocity component is always offshore directed and increases in magnitude as the wave period is increased.

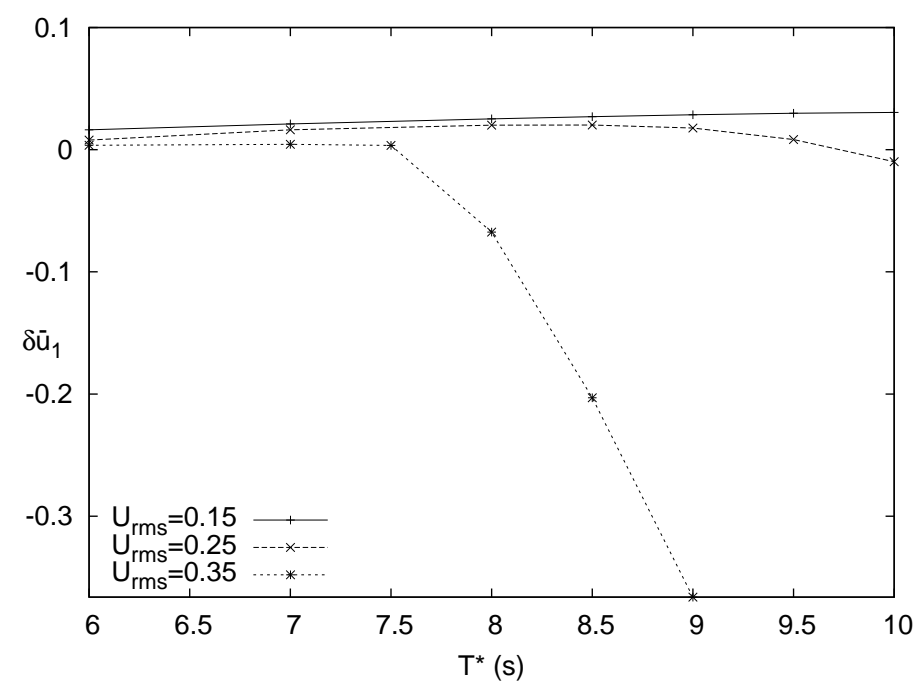

Figure 7. Dimensionless steady velocity component generated by surface waves at the edge of the boundary layer, plotted versus $T^{*}$ for a rippled bed $\left(A_{u}=0.2, d^{*}=0.2 \mathrm{~mm}\right)$. The ripple characteristics are obtained using Soulsby \& Whitehouse's predictor (2005) and are given in Table 1.

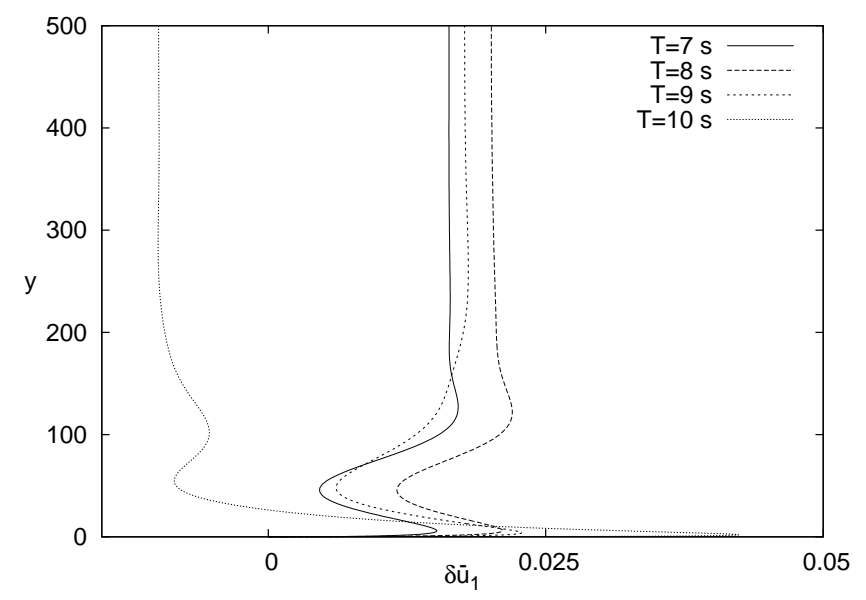

Figure 8. Dimensionless steady velocity component generated by a surface wave, for different values of the wave period $T^{*}$ plotted versus $y$ for a rippled bed $\left(A_{u}=0.2, U_{r m s}=0.25 \mathrm{~m} / \mathrm{s}, d^{*}=0.2\right.$ mm). The ripple characteristics are obtained using Soulsby \& Whitehouse' predictor (2005) and are given in Table 1.

Higher values of $U_{r m s}^{*}\left(U_{r m s}^{*}=0.35 \mathrm{~m} / \mathrm{s}\right)$ lead to negative (shore-ward directed) velocity 
component, which increases in modulus as the wave period is increased. Figure 8 shows the velocity profiles for $U_{r m s}^{*}=0.25 \mathrm{~m} / \mathrm{s}$. It can be noticed that the modulus of the velocity shows one maximum for values of $T^{*}$ ranging in $(6,8.5) \mathrm{s}$, i.e. for the values of $T^{*}$ which lie in the growing part of the curve shown in figure 7. On the other hand, for values of $T^{*}$ which lie in the decreasing part of the curve, two maxima are observed.

\begin{tabular}{|c|c|c|c|c|c|c|}
\hline \multirow[b]{2}{*}{$T^{*}$ [s] } & \multicolumn{2}{|c|}{$U_{r m s}=0.15 \mathrm{~m} / \mathrm{s}$} & \multicolumn{2}{|c|}{$U_{r m s}=0.25 \mathrm{~m} / \mathrm{s}$} & \multicolumn{2}{|c|}{$U_{r m s}=0.35 \mathrm{~m} / \mathrm{s}$} \\
\hline & $\eta_{r}[\mathrm{~cm}]$ & $l_{r}[\mathrm{~cm}]$ & $\eta_{r}[\mathrm{~cm}]$ & $l_{r}[\mathrm{~cm}]$ & $\eta_{r}[\mathrm{~cm}]$ & $l_{r}[\mathrm{~cm}]$ \\
\hline 6 & 3.09 & 20.60 & 3.19 & 21.28 & 2.76 & 18.60 \\
\hline 7 & 3.23 & 21.50 & 3.04 & 20.26 & 2.36 & 17.04 \\
\hline 8 & 3.27 & 21.81 & 2.85 & 19.08 & 2.12 & 16.35 \\
\hline 8.5 & 3.27 & 21.79 & 2.73 & 18.48 & 1.88 & 15.71 \\
\hline 9 & 3.25 & 21.69 & 2.60 & 17.91 & 1.65 & $\overline{15.14}$ \\
\hline 9.5 & 3.23 & 21.51 & 2.45 & 17.36 & 1.43 & 14.62 \\
\hline 10 & 3.19 & 21.28 & 2.29 & 16.84 & 1.24 & 14.15 \\
\hline
\end{tabular}

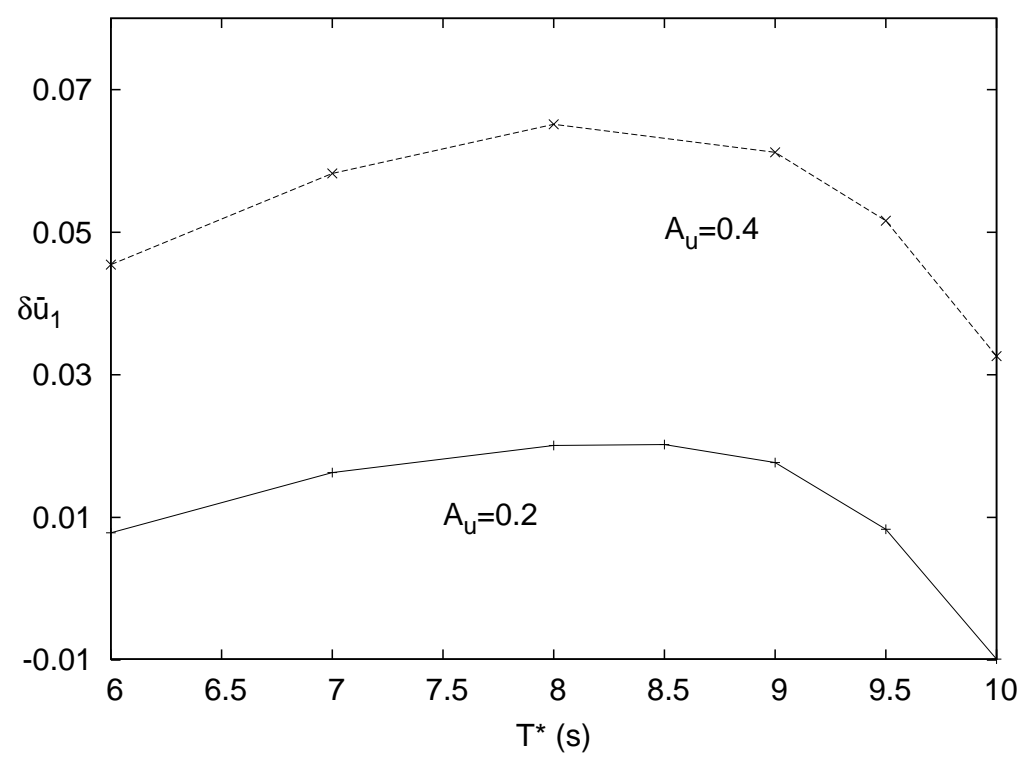

Figure 9. Dimensionless steady velocity component generated by surface waves at the edge of the boundary layer, plotted versus $T^{*}$ for a rippled bed $\left(U_{m s}=0.25 \mathrm{~m} / \mathrm{s}, d^{*}=0.2 \mathrm{~mm}\right)$. The ripple characteristics are obtained using Soulsby $\&$ Whitehouse's predictor (2005).

Figure 9 shows that as $A_{u}$ is increased, the steady velocity component increases in intensity and, for the range of the parameters presently investigated, is offshore directed. Figure 10 shows the influence of the bottom roughness. Indeed the values of the parameters of the runs shown in the figure have been chosen in such a way that ripples are present at the bed. From table 2, which shows the characteristics of the predicted ripples, it can be seen that as $d^{*}$ is increased, both ripple length and height increase. For increasing bottom roughness, the steady velocity component at the edge of the boundary layer 
decreases. Indeed for small values of $d^{*}$, the steady velocity component is off-shore directed, while for values larger than about $0.27 \mathrm{~mm}$, the direction reverses and the magnitude of the steady streaming increases.

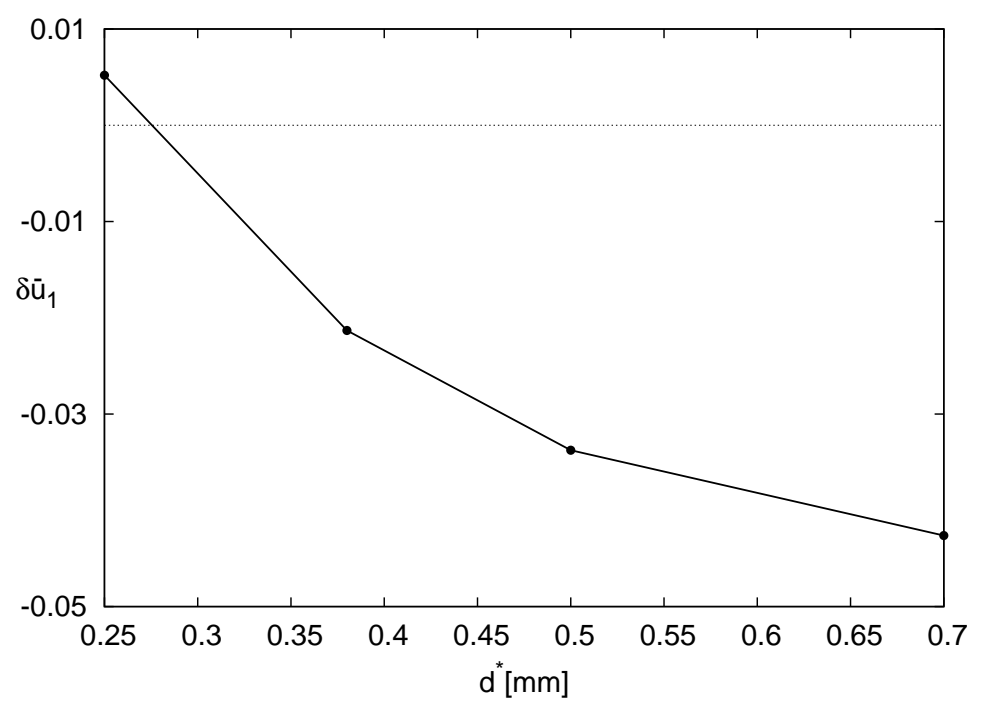

Figure 10. Dimensionless steady velocity component generated by surface waves at the edge of the boundary layer, plotted versus $d^{*}$ for a rippled bed $\left(A_{u}=0.2, U_{m s}=0.25 \mathrm{~m} / \mathrm{s}, T^{*}=7 \mathrm{~s}\right)$. The ripple characteristics are obtained using Soulsby $\&$ Whitehouse's predictor (2005) and are given in Table 2.

\begin{tabular}{|c|c|c|}
\hline \multicolumn{3}{|c|}{$\begin{array}{l}\text { Table 2: Ripple height }\left(\eta_{r}\right) \text { and wavelength }\left(l_{r}\right) \text { as } \\
\text { function of the wave period } T^{\star} \text { for } \\
A_{u}=0.2, U_{r m s}=0.25 m / s, \mathrm{~T}=7 \mathrm{~s}\end{array}$} \\
\hline$d^{\star}[\mathrm{mm}]$ & $\eta_{r}[\mathrm{~cm}]$ & $l_{r}[\mathrm{~cm}]$ \\
\hline 0.25 & 4.05 & 26.97 \\
\hline 0.375 & 5.92 & 39.47 \\
\hline 0.5 & 6.84 & 45.58 \\
\hline 0.7 & 7.54 & 50.24 \\
\hline
\end{tabular}

\section{CONCLUSIONS}

For large values of the sediment mobility number, such that small-scale bedforms (ripples) are washed out, sea waves appear to induce a net (wave-averaged) sediment transport which is in the onshore direction even though the wave-averaged velocity points in the onshore/offshore direction depending on the wave characteristics and the local water depth. This qualitative finding is supported by experimental observations (e.g. Schretlen, Ribberink \& O'Donoghue 2010). In the range of the sediment mobility number presently investigated, the suspended load prevails on the bed load and the behaviour of steady part of the suspended load transport differs from that of the velocity.

When ripples are present, the model predicts their characteristics by using Soulsby \& Whitehouse's 
predictor (2005). The results have shown that, for intermediate values of $U_{r m s}^{*}$, an increase on the wave period, once the other parameters are kept fixed, reverts the direction of the steady streaming at the edge of the boundary layer from offshore directed to onshore directed. For the largest values of $U_{r m s}^{*}$, the dimensionless steady streaming is found to be onshore-directed while the smaller values of $U_{r m s}^{*}$ give rise to shore-ward directed streaming. An increase of the sediment diameter, which affects the ripple characteristics, causes a decay of the offshore-directed steady streaming and eventually an inversion of the steady flow.

\section{ACKNOWLEDGMENTS}

This study is founded by the "Ministero dell'Istruzione, dell'Università e della Ricerca' under the research project 2008YNPNT9-003 "Idrodinamica e morfodinamica nella regione dei frangenti".

\section{REFERENCES}

Blondeaux P. and Colombini M. 1985. Pulsatile turbulent pipe flow. Proceedings 5th International Symposium on Turbulent Shear Flows Ithaca (NY) (ed. J. L. Lumley, B. E. Launder, N. C. Reynolds \& J. A. Whitelaw).

Blondeaux, P. 1987 Turbulent boundary layer at the bottom of gravity waves. J. Hydraul. Res. 25 (4), 447-464.

Blondeaux, P. \& Vittori, G. 1991a Vorticity dynamics in an oscillatory flow over a rippled bed.

J. Fluid Mech. 226, 257-289.

Blondeaux, P. \& Vittori, G. 1991b A route to chaos in an oscillatory flow: Feigenbaum scenario. Phys. Fluids A 3, 2492-2495.

Blondeaux, P., Vittori, G. \& Foti, E. 2000 Migrating sea ripples. Eur. J. Mech. (B/Fluids) 19, 285-301.

Blondeaux P., Vittori G., Bruschi A., Lalli F. and Pesarono V. 2012. Steady streaming and sediment transport at the bottom of sea waves. Journal of Fluid Mechanics, 697,115-149 doi:10.1017/jfm.2012.50

Collins, J. I. 1963 Inception of turbulence at the bed under periodic gravity waves. J. Geophys. Res. 68, 6007-6014.

Chowdhury, S. A., Sato, M. \& Ueno, A. 1997 Numerical model of the turbulent wave boundary layer induced by finite amplitude water waves. Appl. Ocean Res. 19, 201-209.

Collins, J. I. 1963 Inception of turbulence at the bed under periodic gravity waves. J. Geophys. Res. 68, 6007-6014.

Fredsoe J. and Deigaard R. 1992. Mechanics of Coastal Sediment Transport. Advance Series on Ocean Engineering, vol. 3, World Scientific.

Gonzalez-Rodriguez, D. \& Madsen, O. S. 2011 Boundary layer hydrodynamics and bed load sediment transport in oscillating water tunnels. J. Fluid Mech. 667, 48-84.

Hsu, T. W. \& Ou, S. H. 1994 On the mass transport of water waves. Ocean Engng 21 (2), 195-206.

Justesen, P. A. 1988 Prediction of turbulent oscillatory flow over rough beds. Coastal Engineering

12, 257-284.

Kajiura, K. 1968 A model of the bottom boundary layer in water waves. Bull. Earthq. Res. Inst.

46, 75-123.

Longuet-Higgins, M. S. 1953. Mass transport in water waves. Phil. Trans. R. Soc. Lond. A 245, 535581.

Longuet-Higgins, M. S. 1958 The mechanism of the boundary layer near the bottom in a progressive wave. In Proceedings of the 6th International Conference on Coastal Engineering, Berkeley (CA), ASCE, 184-193.

Mei, C. C. 1989. The Applied Dynamics of Ocean Surface Waves. Advanced Series on Ocean Engineering, vol. 1, World Scientific.

Saffman P.G. 1970. A model for inhomogeneous turbulent flow. Proc. R. Soc. Lond. A 317, 417-433.

Saffman P. G. and Wilcox P. C. 1974. Turbulence model predictions for turbulent boundary layers. AIAA J. 12, 541-546.

Scandura P. and Foti E. 2011. Measuerements of wave-induced steady currents outside the surf zone.

Journal of Hydraulic Research, 49, 64-71

Sleath J.F.A, 1984. Sea bed mechanics. Wiley

Schretlen, J. J. L. M., Ribberink, J. S. \& O’Donoghue, T. 2010. Boundary layer flow and 
sand transport under full scale surface waves. In Proceedings of the 32nd International Conference on Coastal Engineering, Shangai 2010 (ed. J. M. Smith \& P. Lynett). Coastal Engineering Research Council.

Soulsby R.L., Whitehouse R.J.S. 2005. Prediction of ripple properties in shelf seas. Report TR150. HR Wallingford.

Trowbridge, J. \& Madsen, O. S. 1984a Turbulent wave boundary layers. 1. Model formulation

and first-order solution. J. Geophys. Res. 89 (C5), 7989-7997.

Trowbridge J. \& Madsen O. S. 1984b. Turbulent wave boundary layers. 2. Second-order theory and mass-transport. J. Geophys. Res. 89 (C5), 7999-8007.

Van Der Werf, J. J., Schretlen, J. J. L. M., Ribberink, J. S. \& O'Donoghue, T. 2009. Database of fullscale laboratory experiments on wave-driven sand transport processes. Coastal Engineering 56 (7), 726-732.

Van Doorn, T. 1981 Experimental investigation of near-bottom velocities in water waves with and without a current. Rep. MI. 423 Delft Hydraulics Lab.

Van Rijn L. 1991. Sediment transport in combined waves and currents. Proceedings Euromech 262 Colloquium Sand Transport in Rivers, Estuaries and the Sea, Wallingford (UK) (ed. R. Soulsby \& R. Bettess). A.A. Balkema.

Vittori G. and Blondeaux P. 1996 Mass transport under sea waves propagating over a rippled bed. $J$. Fluid Mech. 314, 247-265.

Zyserman, J. A. and Fredsoe J. 1994. Data analysis of bed concentration of suspended sediment. Hydraul. Engng 120 (9), 1021-1042. 